\title{
Using Blockchain for Business \& Marketing Improvement
}

\author{
Panagiotis Gatomatis \\ University of Patras
}

\author{
Konstantinos Tsiomos \\ Blockchain 2050 BV
}

\author{
Nikos Bogonikolos \\ Aratos Systems BV
}

\begin{abstract}
In the $21^{\text {st }}$ century's global business environment, there is a growing demand for credibility. Recent research has shed light on the global decline in trusting brands. Typically, trusted third parties have intervened to ensure the trust required for different business transactions. This paper examines the impact of Blockchain technology on business and marketing from a variety of perspectives. Conclusions are drawn regarding the anticipated course of business and marketing advances as a consequence of these technological advancements, culminating in the widely held belief that Blockchain innovations have the potential to fundamentally transform the economy, culture, political structures, and research activities in positive ways.
\end{abstract}

Keywords: blockchain, business, marketing

\section{INTRODUCTION}

More and more industry analysts agree that Blockchain technology would have a significant impact on the existence of businesses, including the way they are financed and run, how they generate value, and how they conduct basic functions including marketing, accounting, and motivating employees and customers. Many management functions are likely to be replaced by software in some situations [1].

Blockchain technology provides companies with a bright new capability for sellers and buyers to store and trade value through a secure network, ledger, or database without the need for conventional intermediaries.

Values aren't saved in local repositories; instead, they're represented by transactions in distributed spreadsheets or ledgers, which takes advantage of a massive peer-to-peer network's resources for the validation and authorization transactions.

A blockchain has a wealth of advantages, the majority of which are well-known today. As a result, we'll focus on three of them, which we believe are more important to the case at hand. For start, it is distributed because it operates on remote nodes all over the world, eliminating the need for a central database, which makes it impossible to be hacked. It can be opened for the public: since it's based on a global peer-to-peer 
network, everyone can have access to it at any given time. And last, but not least, it is secure because it employs high-level encryption techniques to ensure confidentiality of data.

\section{THE METHODOLOGY OF BLOCKCHAIN TECHNOLOGY}

We can summarize Blockchain in a few words: a digital ledger in the form of a distributed database that is shared through a wide computer network. Each of the network's nodes has a copy of these shared ledgers. Each block in the chain constitutes a set of data that has been encrypted with each block having to undergo through a series of consensus procedures before it can be validated and connected to the chain. The blockchain has no design flaws due to its complex and highly encrypted nature which renders hackers unable to crack or hack a node to steal data or tamper with them. To achieve such goal, the attackers should target every node on the chain at the same time, something that would require unrealistic amount of resources. As a result, a blockchain is practically un-hackable.

A Blockchain network continuously verifies, clears, and stores transactions in the form digital datablocks that are linked to previous blocks, forming a chain. For a block to be valid, it must first refer to the one before it. This mechanism applies permanent timestamps and records transactions, preventing tampering with the shared ledger. An interference or alteration of data would require the attacker to completely rewrite the blockchain's entire transaction history. The blockchain's security and reliability are ensured by collective self-interest. As a result, Blockchain serves as a useful and valuable tool for safely and securely shattering conventional and centralized structures.

Thus, it becomes apparent that this technology has the transformative power to pave the way for "an internet of value" [2], in which buyers and sellers can directly store and trade valuable assets without the need for intermediaries.

\section{THE BUSINESS ENVIRONMENT INDUCED TRANSITION}

According to studies, Blockchain technology is expected to present soon groundbreaking impacts on a global scale. In a recent World Economic Forum, it was estimated that a significant portion of global GDP will eventually be stored on blockchain, with predictions estimating a $10 \%$ by 2027 [3]. While this is major news, it raises the question of how much Blockchain technology will impact the global economy. The so far indications show that this will be done to a great extent.

In the coming years, blockchain technology is expected to have a significant effect on all major business sectors, with great potential to disrupt the global economy on a wide scale. In fact, such the disruption has already begun. Below, we examine six cases which blockchain technology has already begun to affect:

\section{General Management}

It is a very simple concept to grasp that Blockchain is changing the way companies are structured and run because it enables businesses to eliminate transaction costs and use external resources as effectively as internal resources. In certain cases, vertical integration can continue to make sense (e.g. for manufacturingcontrolled products, or wherever companies have industry-leading strengths throughout the supply chain). However, blockchain-based networks would be best suited in the vast majority of cases for developing goods and services, as well as providing value to stakeholders.

\section{Financial Management}

Financial management will benefit from the concepts of blockchain technology, which were created to combat banking fraud. Businesses may use blockchain-based systems to monitor and control their budget spending from start to finish. This reduces the risk of being overcharged for something minor concealed in a chain of many transactions, as well as ensuring better long-term results. Financial executives will benefit from better security by implementing blockchain technology, ensuring that their ROI will not be harmed. 


\section{Business Accounting}

The use of blockchain technology simplifies the life of an accountant. With blockchain, the risk of fraud is reduced, control is increased, obfuscated data is reduced, and data transparency is increased. Since transactions are registered in a non-editable way, blockchain technology provides absolute transparency and anonymity. This is a game-changer in terms of financial bookkeeping, maintenance, and auditing.

\section{Human Resources Management}

The hiring processes will soon be revolutionized thanks to blockchain technology, which will allow human resources personnel to verify applicants and current employees much more quickly and efficiently since third-party interference with faulty information on work applicants will no longer be a problem. Payroll would now be simplified, even for multinational firms and businesses employing foreign workers. More people will invest in blockchain-based cryptocurrencies in the future, rather than tying it to their home country's currency and banks, which is expected to change how people save for retirement.

\section{Sales Marketing}

A blockchain would be able to tell you about individuals or companies that are potential clients, just as it can tell you about potential contractors and partners.

\section{IT and Cybersecurity}

Cybersecurity is by far the most important aspect of the modern global economy, and without it, the economy can be easily compromised, disrupted, and, in certain cases, damaged. Blockchain has the potential to strengthen this vital sector of the economy due to the fact that it can securely and efficiently monitor digital transactions and interactions, providing accountability and security to build a solid framework on which businesses can rely. Businesses may use blockchain to improve their security: not only they may conduct transactions but also store data in a way that ensures the integrity of the transactions and makes their data permanent.

\section{THE BENEFITS OF BLOCKCHAIN TECHNOLOGY IN BUSINESS \& MARKETING}

Blockchain is a decentralized peer-to-peer network that does not have a centralized authority. The decentralized concept's fundamental idea provides a plethora of advantages for businesses [4]. It provides them with the following advantages at its heart:

\section{Distributed}

Business organizations will greatly benefit from decentralization thanks to blockchain. Business entities may use blockchain to run their processes in a distributed environment. Since a distributed architecture does not need a centralized database, they can either own or volunteer the necessary computers (nodes). Each peer has its own ledger, preventing malicious actors from altering the data.

\section{Public}

Business organizations are always keen to connect with their users. This means that they always have to have a particular process open to the end-user so that they can provide feedback or check information whenever needed. Blockchain supports business organizations do just that: create a federated blockchain network, as a mix of the public and private networks. The business organization will keep its critical processes and data in their private network while making proper use of the public features of the network. Thus, any user can check the information on the network anytime. This builds end-users trust and also makes business organizations accountable for their actions and deeds.

\section{Security}

Businesses are concerned with the protection of their trade secrets because they do not want their confidential information to be leaked. Their critical data are more secure thanks to blockchain. To ensure 
the data always remains secure, blockchain employs high-level encryption. Many businesses often deploy a fully private encrypted blockchain network to ensure that their data is never accessed by someone who isn't allowed to do so.

\section{Transaction Cost}

Blockchain is cost-effective since it is not reliant on a single agency. When opposed to a centralized method, the cost of handling a transaction is comparably lower. Furthermore, since the data is not stored in a centralized database, everyone in the business enterprise can access it from any location they want, eliminating the need to hop between conversations requesting the information. The transaction cost can be absolutely eliminated in some situations, ensuring proper growth and performance.

\section{No Intermediaries}

Business entities no longer have to be concerned with third-party solutions that serve as service intermediaries. This relieves business entities of a significant amount of work, but it also exposes them to unnecessary risk. For example, if one of the intermediate servers fails, the entire work process can come to a halt and the business company will lose a lot of value if there is no backup.

\section{THE DECENTRALIZED AUTONOMOUS ORGANIZATION (DAO) CONCEPT}

Given the above, it is obvious that blockchain can have a huge impact on business architecture. The emphasis has shifted away from a stale hierarchical/centralized approach and towards a more decentralized approach. From now on, the design concept would be focused on a Decentralized Autonomous Organization (DAO). As a result of using DAO, businesses become more independent and competitive, while also changing their structure [5].

DAOs use a mix of distributed ledger technology, smart contracts, connected devices and analytics to operate independently. To do so, they implement properly developed autonomous software that includes immutable code, and each of the actors is properly placed in the DAO according to their position and role. Because of the immutability of Blockchain, it's important to remember that once the rules are set, they can't be changed. This is essential in order to give the business organization autonomy. It also allows the various actors to build and maintain trust among them. The consensus algorithm can guarantee that and also protects data validity and immutability.

In a DAO, power is determined by an actor's level of trustworthiness. Since there is no hierarchy, a trust-based approach is required. Depending on its actions and behavior, a node gains trust. If it has a high level of trust, it can serve as a network decision-maker. As a result, the power within the business organization is equitably distributed. The actors' power is determined by their degree of trust, the number of tokens they have, and their achievements. Each actor can change the power balance by including three elements in their power calculations.

Aside from the power change, business organization design is also impacted. Cryptography, for example, is used to establish trust. Traditional decentralized organizations make decisions based on seniority and experience, while in the DAO, smart contracts are used to make decisions automatically. The governance in DAO is also embedded in code. For traditional decentralized organizations, the board of directors is in charge of it.

\section{CONCLUSIONS}

It is obvious that the revolution occurring in the organizational conditions of business organizations has in extend and impact, not only in terms of decision-making and operating conditions but also in terms of financial transaction methodology and security alike. If we keep track of the sectors of business activities that have been affected, we will realize that the list is long. It is clear that blockchain technology has the ability to transform a broad range of business processes. As this technology matures and establishes itself in business processes, process experts may find it necessary to ensure that the value generated comes from 
exploring the opportunities this technology can allow in business processes rather than simply replacing existing technologies with new ones. Blockchain, in general, seems to be changing everything in the business world. It possesses an immense amount of potential to implement new and improved methodologies and ways to make the business ecosystem more secure, trustworthy, transparent, and efficient.

\section{REFERENCES}

Hileman, G. (2016, June 15). State of Blockchain Q1 2016: Blockchain Funding Overtakes Bitcoin. CoinDesk. Retrieved from https://www.coindesk.com/state-of-blockchain-q1-2016

Korizky, R. (2105, September 26). World Economic Forum Survey: 10\% of global GDP may be stored with blockchain technology by 2027. (COINFOX). Retrieved from

https://www.coinfox.info/news/3184-world-economic-forum-survey-10-of-global-gdp-may-bestored-with-blockchain-technology-by-2027

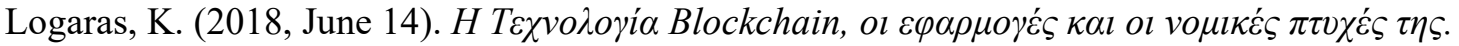
Lawspot.gr. Retrieved from https://www.lawspot.gr/nomika-nea/h-tehnologia-blockchain-oiefarmoges-kai-oi-nomikes-ptyhes-tis

Tapscott, D., \& Tapscott, A. (2016, December 7). How Blockchain Will Change Organizations. MIT Sloan Management Review (MIT SMR). Retrieved from https://sloanreview.mit.edu/article/howblockchain-will-changeorganizations/?utm_source=author\&utm_medium=referral\&utm_campaign=smr-marketing

Wikipedia contributors. (2021, May 2). Decentralized Autonomous Organization. Wikipedia, The Free Encyclopedia. Retrieved from https://en.wikipedia.org/w/index.php?title=Decentralized_autonomous_organization\&oldid=1021 087355 